\title{
Detection of mullerian duct anomalies: diagnostic utility of two dimensional ultrasonography as compared to magnetic resonance imaging
}

\author{
Krishna Pratap Singh Senger ${ }^{1}$, Ajay K. Singh ${ }^{1}$, Vivek Sharma1, Ankita Singh ${ }^{2}$, R. Ravikumar \\ ${ }^{1}$ Department of Radiodiagnosis and Imaging, Army Hospital, Research and Referral, New Delhi, India \\ ${ }^{2}$ Research Consultant, Former Research Associate, Intrahealth International, BMGF, Lucknow, Uttar Pradesh, India \\ ${ }^{3}$ Commandant, Military Hospital, Bhopal, Madhya Pradesh, India
}

Received: 24 November 2016

Revised: 28 November 2016

Accepted: 29 November 2016

\section{*Correspondence: \\ Dr. Krishna Pratap Singh Senger, \\ E-mail: drkpss009@gmail.com}

Copyright: ( ) the author(s), publisher and licensee Medip Academy. This is an open-access article distributed under the terms of the Creative Commons Attribution Non-Commercial License, which permits unrestricted non-commercial use, distribution, and reproduction in any medium, provided the original work is properly cited.

\section{ABSTRACT}

Background: Mullerian duct anomalies (MDAs) are a fascinating group of disorders that have varied clinical presentation from being asymptomatic to primary amenorrhea to inability to reproduce. Correct diagnosis of the condition plays a crucial role in management. Imaging plays a pivotal role in making correct diagnosis. This study aims to find the prevalence of MDAs amongst study population and their relation with infertility and also compares diagnostic utility of pelvic ultrasound with MRI.

Methods: A randomized diagnostic test evaluation study was conducted in the Department of Radiodiagnosis and Imaging of a tertiary care teaching hospital over a period of 2 years. The patient first underwent pelvic 2D USG in multiple planes using curvilinear probe of $3 \mathrm{MHz}$ to $5 \mathrm{MHz}$. frequency and then MRI.

Results: Most common MDA in total study sample and in primary infertility group is arcuate uterus while in recurrent abortions group it is unicornuate uterus. Out of total study sample of 75 patients 2D USG detected 18 cases of MDA while MRI detected 22 cases of MDA. So, 2D USG failed to detect 04 cases of MDA in total study population bringing overall sensitivity of 2D USG as $81.8 \%$, specificity of $100 \%$, PPV of $100 \%$, NPV of $93.4 \%$ and accuracy of $94.6 \%$.

Conclusions: 2D USG has a few limitations but in view of relatively simple imaging procedure, ease of availability and cost effectiveness it should be utilized as an initial imaging modality in patients with suspicion of MDAs.

Keywords: Infertility, Mullerian duct anomalies, MRI, Uterus, 2D ultrasonography

\section{INTRODUCTION}

Mullerian duct anomalies are a fascinating group of disorders that have varied clinical presentation from being asymptomatic to primary amenorrhoea, inability to conceive, recurrent abortions, abnormal foetal lie and preterm labor. The disorder is usually recognised once the patient fails to achieve menarche or has problem in conceiving or presents with repeated abortions. The psychosocial impact of these disorders is tremendous as far as their clinical outcome is concerned.

A meta-analysis done in United Kingdom by University of Sheffield, suggests that the prevalence of Mullerian duct anomalies is $6.7 \%$ [95\% Confidence interval (CI), (6.0-7.4) in the general population, $7.3 \%$ (95\% CI, 6.77.9) in infertile female population and $16.7 \%$ (95\% CI, 14.8-18.6) in recurrent miscarriage population. ${ }^{1-3}$ 
Correct diagnosis of the condition plays a pivotal role in management as the plan of management varies with the diagnosis. Imaging plays an important role as with the availability of advanced high resolution ultrasonography machines and MRI, invasive diagnostic approach like laparoscopy and hysteroscopy are reserved only for few cases where diagnosis is uncertain even after extensive imaging. 4,5

Initial imaging evaluation of patients is done with $2 \mathrm{D}$ transabdominal USG (2D USG) which helps in identifying MDAs in clinically suspicious cases. The findings of 2D USG are confirmed using MRI which helps to confirm the diagnosis and may even sub classify it. Various studies have shown high sensitivity and specificity of 3D USG in diagnosing MDAs, but due to its limited availability it is remains underutilised. ${ }^{6-9}$

Previous studies have shown that two dimensional transabdominal ultrasonography (2D USG) has high specificity coupled with low sensitivity. When used in isolation 2D USG is able to identify major uterine anomaly easily but it may not be able to sub classify it in all cases due to its imaging restrictions. ${ }^{11}$ Sometimes it may entirely miss a Mullerian duct anomalies due to limitations mentioned above. Even though 2D USG has a few limitations but in view of relatively simple imaging procedure, ease of availability and cost effectiveness it should be utilised as an initial imaging modality in patients with suspicion of MDAs.

In view of above, a prospective diagnostic study was envisaged to look for the prevalence of MDAs in our study population, to correlate MDAs with various aspects of reproductive potential. Also to evaluate the diagnostic utility of two dimensional ultrasonography in detecting Mullerian Duct Anomalies as compared to Magnetic Resonance Imaging (MRI). This study is unique and the only study till date taking into account the entire spectrum of Mullerian duct anomalies across all age groups in one go.

\section{METHODS}

This study was a diagnostic test evaluation study conducted in the Department of Radiodiagnosis and Imaging of a tertiary care teaching hospital in association with the Department of Obstetrics and Gynecology over a period of 2 years. The study population comprised of 75 female patients aged between 17 to 40 years with mean age of 26 years. The inclusion criteria were designed to include entire spectrum of clinical presentation of MDAs i.e., patients of primary infertility, primary amenorrhea and patients with recurrent miscarriages. Ethical clearance was taken from institutional ethical committee. To achieve random sampling, every third patient reporting to the Department of Radiodiagnosis and Imaging after referral from Obstetrics and Gynecology OPD and fulfilling the required inclusion criteria were considered for inclusion in the study.
The patient was given a detailed informed about the imaging modalities and their safety profile and a written informed consent was taken before being taken up for imaging. The patient is subjected to pelvic ultrasound through two dimensional transabdominal route in an adequately full urinary bladder on a day which corresponds to secretary phase i.e., 15-20th day of her menstrual cycle in patients with regular normal cycles. Those patients who had irregular periods imaging is still done in secretory phase and the calculation done on the basis of length of her average menstrual cycle. The pelvic imaging is done in multiple planes using curvilinear probe at a frequency of $3 \mathrm{MHz}$ to $5 \mathrm{MHz}$ (Table 1 shows various parameters used during 2D USG scan of the pelvis).

After 2D USG patients underwent MRI without contrast administration on the same day. The equipment used was 1.5 Tesla (Symphony, Siemens Medical Systems, Erlangen, Germany) MR system with surface coils for better signal to noise ratio (SNR). The localisers were taken in all three planes and first sequence acquired was T2 Sagittal, followed by T2 Coronal along true coronal plane of uterus. T2 coronal along true coronal plane was the most useful sequence because the fundal contour was best appreciated in this sequence which is very important to differentiate various MDAs. Finally T1 and T2 axial images were obtained (Scanning parameters used during MRI examination of the pelvic region are shown in Table 2).

All the images (both 2D USG and MRI) were shown in a common forum to a group of radiologists and consensus regarding findings and final diagnosis is reached after thorough discussion within the group. The Mullerian duct anomalies are then classified as per American Fertility Society (AFS) based on the consensus of the forum.

Diagnostic criteria for differentiating various MDAs by 2D USG and MRI: Configuration of external fundal contour is very important to distinguish various sub types of MDAs mainly septate, bicornuate, didelphys and arcuate uterus. The criteria used in our study are:

1. Deep fundal cleft $(>1 \mathrm{~cm})$ below inter-cornual line is suggestive of bicornuate/didelphys. Intercornual distance $>4 \mathrm{~cm}$ is suggestive of bicornuate uterus. ${ }^{5,12}$

2. Deep fundal cleft $(>1 \mathrm{~cm})$ with duplicated uterine horns, cervix and vagina is suggestive of uterus didelphys. On the other hand bicornuate uterus shows union in lower part of uterus/cervix.

3. Smooth fundal contour or small fundal depression $(<1 \mathrm{~cm})$ is seen in septate uterus. Uterine cavity is bifid on axial images.

4. Unicornuate uterus shows a curved and slender uterine horn without the usual rounded fundal contour, and the single horn is usually laterally deviated.

5. A hypoplastic uterus is diagnosed on the basis of its small size and reduced intercornual distance $(<2 \mathrm{~cm})$. 
The zonal anatomy may be poorly differentiated in T2-weighted images of hypoplastic uterus.

6. In agenesis of the mullerian duct derivatives no identifiable uterine tissue or the upper part of the vagina could be seen.

A deep fundal cleft greater than $1 \mathrm{~cm}$ has been reported to be $100 \%$ sensitive and specific in differentiation of fusion anomalies (didelphys and bicornuate) from reabsorption anomalies (septate and arcuate uterus). ${ }^{12}$

The data was compiled and statistically analysed using statistical software SPSS version 17 and Epi Info Version 3.3.2. Chi square and Fisher Exact test (test of significance) were used to determine statistical significance.

\section{RESULTS}

Table 1: Scanning parameters used during two dimensional transabdominal scanning of pelvic region.

\begin{tabular}{|ll|}
\hline Parameters & Measure \\
\hline Frequency & $3 \mathrm{MHz}-5.0 \mathrm{MHz}$ \\
\hline Gain & $50-80 \%$ \\
\hline D (Depth) & $10-30 \mathrm{~cm}$ \\
\hline DR (Dynamic range) & $70-90$ \\
\hline FR (Frame rate) & $26 \mathrm{~Hz}$ \\
\hline
\end{tabular}

A total sample consisting of 75 patients were included in the study after satisfying the inclusion criteria. The study sample comprised of 11 Patients with Primary amenorrhoea, 64 patients of infertility. Of these 64 patients, 48 patients presented with Primary Infertility and 16 patients presented with recurrent abortions.

Table 2: Scanning parameters used during MRI scan of the pelvic region.

\begin{tabular}{|lllll|}
\hline Parameters & $\begin{array}{l}\text { T2 } \\
\text { Sag }\end{array}$ & $\begin{array}{l}\text { T2 } \\
\text { Cor }\end{array}$ & $\begin{array}{l}\text { T2 } \\
\text { Axial }\end{array}$ & $\begin{array}{l}\text { T1 } \\
\text { Axial }\end{array}$ \\
\hline TR $(\mathrm{mSec})$ & 4930 & 4000 & 3060 & 459 \\
\hline TE $(\mathrm{mSec})$ & 100 & 100 & 100 & 13 \\
\hline Voxel size & $\begin{array}{l}1 \times 0.9 \\
\text { x } 5\end{array}$ & $\begin{array}{l}1 \times 0.9 \\
\text { x 5 }\end{array}$ & $\begin{array}{l}1 \times 0.9 \\
\text { x 5 }\end{array}$ & $\begin{array}{l}1 \times 0.9 \\
\text { x 5 }\end{array}$ \\
\hline Distance factor & 0 & 0 & 0 & 0 \\
\hline FoV (mm) & 230 & 240 & 231 & 231 \\
\hline $\begin{array}{l}\text { Slice thickness } \\
\text { (mm) }\end{array}$ & 5 & 5 & 5 & 5 \\
\hline $\begin{array}{l}\text { Time of scan } \\
\text { (min) }\end{array}$ & 2.44 & 2.22 & 2.07 & 2.01 \\
\hline
\end{tabular}

Out of these 11 cases of primary amenorrhoea, on USG examination Mullerian duct anomalies were noted in 06 patients (uterine agenesis in 02 patients, uterine hypoplasia in 02 patients, vaginal agenesis with bicornuate uterus in 01 case and uterus didelphys in 01 case), 05 cases had normal uterine anatomy. On MRI findings were confirmed and there is no discrepancy in findings between 2D USG and MRI (Table 3).

Table 3: Primary amenorrhoea division of cases into subtypes.

\begin{tabular}{|l|ll|}
\hline Anomaly & MRI & 2D-USG \\
\hline Uterine agenesis & 2 & 2 \\
\hline Uterine hypoplasia & 2 & 2 \\
\hline $\begin{array}{l}\text { Vaginal agenesis + Bicornuate } \\
\text { uterus }\end{array}$ & 1 & 1 \\
\hline Uterus didelphys & 1 & 1 \\
\hline Normal appearance & 5 & 5 \\
\hline Total & 11 & 11 \\
\hline
\end{tabular}

Table 4: Primary infertility division of cases into subtypes.

\begin{tabular}{|lll|}
\hline Anomaly & MRI & 2D-USG \\
\hline Arcuate uterus & 5 & 4 \\
\hline Septate uterus & 1 & 0 \\
\hline Bicornuate uterus & 2 & 3 \\
\hline Unicornuate uterus & 2 & 1 \\
\hline Uterus didelphys & 0 & 0 \\
\hline Miscellaneous & 13 & 13 \\
\hline Normal appearance & 25 & 27 \\
\hline Total & 48 & 48 \\
\hline
\end{tabular}

Table 5: Recurrent abortions division of cases into subtypes.

\begin{tabular}{|lll|}
\hline Anomaly & MIRI & 2D-USG \\
\hline Arcuate uterus & 1 & 0 \\
\hline Septate uterus & 2 & 2 \\
\hline Bicornuate uterus & 0 & 0 \\
\hline Unicornuate uterus & 3 & 2 \\
\hline Uterus didelphys & 0 & 0 \\
\hline Miscellaneous & 2 & 2 \\
\hline Normal appearance & 8 & 10 \\
\hline Total & 16 & 16 \\
\hline
\end{tabular}

Table 6: Total study population division of cases into subtypes.

\begin{tabular}{|lll|}
\hline Anomaly & MRI & 2D-USG \\
\hline Uterine agenesis & 2 & 2 \\
\hline Uterine hypoplasia & 2 & 2 \\
\hline Arcuate uterus & 6 & 4 \\
\hline Septate uterus & 3 & 2 \\
\hline Bicornuate uterus & 3 & 4 \\
\hline Unicornuate uterus & 5 & 3 \\
\hline Uterus didelphys & 1 & 1 \\
\hline Miscellaneous & 15 & 15 \\
\hline Normal appearance & 38 & 42 \\
\hline Total & 75 & 75 \\
\hline
\end{tabular}


Amongst 48 patients who presented with primary infertility 2D USG detected 08 cases of MDA (arcuate04, bicornuate-03, and unicornuate-01). On MRI Mullerian duct anomalies are noted in 10 cases (arcuate05, bicornuate-02, unicornuate-02, septate-01), 25 patients were found to have no utero-cervical anomaly and 13 patients had miscellaneous lesions (fibroids-04, ovarian cysts-02, cervicitis-01, adenomyosis-02, pelvic inflammatory disease-01 and PCOD-03). There is discrepancy in findings between 2D USG and MRI (Table 4).

There were 16 patients who presented with recurrent abortions (with 3 or more abortions). On 2D USG only 04 cases of MDA (septate-02, unicornuate-02) were detected. While on MRI, MDA are noted in 06 patients (03-unicornuate, 02-septate and 01-arcuate). 2D USG was unable to detect one unicornuate and one arcuate uterus which were detected by MRI. 08 patients showed normal scan and 02 patients (one patient had multiple uterine fibroids and one patient had complex adnexal cysts bilaterally (Table 5).

Table 7: 2X2 table for total study sample $(n=75)$.

\begin{tabular}{|lll|l|}
\hline & True+ & True- & \\
\hline 2D USG + & 18 & 00 & 00 \\
\hline 2D USG - & 04 & 53 & 57 \\
\hline Total & 22 & 53 & 75 \\
\hline
\end{tabular}

\section{Tests of significance}

Fisher exact test (one tailed): 0.000000; Fisher exact test (two tailed): 0.000000; Yates corrected Chi-square; $\mathrm{p}$ value: 0.000000 .

\section{Screening [95\% CI]}

Sensitivity: $81.8 \%$ [59, 94]; Specificity: $100 \%$ [92, 100]; Accuracy: $94.6 \%$ [86, 98]; PPV: $100 \%$ [78, 100]; NPV: $93.4 \%$ $[82,98]$.

Table 8: Combined sensitivity, specificity, PPV, NPV and accuracy for all sub groups, subtypes and total study population.

\begin{tabular}{|lllllll|}
\hline Sub groups & Cases(n) & Sensitivity (\%) & Specificity (\%) & PPV (\%) & NPV (\%) & Accuracy (\%) \\
\hline Uterine agenesis & 02 & 100 & 100 & 100 & 100 & 100 \\
\hline Uterine hypoplasia & 02 & 100 & 100 & 100 & 100 & 100 \\
\hline Arcuate uterus & 06 & 66.6 & 100 & 100 & 97.2 & 97.3 \\
\hline Septate uterus & 03 & 66.6 & 100 & 100 & 98.6 & 99 \\
\hline Bicornuate uterus & 03 & 100 & 98.6 & 75 & 100 & 99 \\
\hline Unicornuate uterus & 05 & 60 & 100 & 100 & 97.3 & 97 \\
\hline Didelphys uterus & 01 & 100 & 100 & 100 & 100 & 100 \\
\hline Pr. amenorrhoea & 11 & 100 & 100 & 100 & 100 & 100 \\
\hline Pr. infertility & 48 & 80 & 100 & 100 & 95.2 & 96 \\
\hline Recur. abortions & 16 & 66.6 & 100 & 100 & 85.7 & 88 \\
\hline Total inf. population & 64 & 75 & 100 & 100 & 92.8 & 94 \\
\hline Total study sample & 75 & 81.8 & 100 & 100 & 93.4 & 94.6 \\
\hline
\end{tabular}

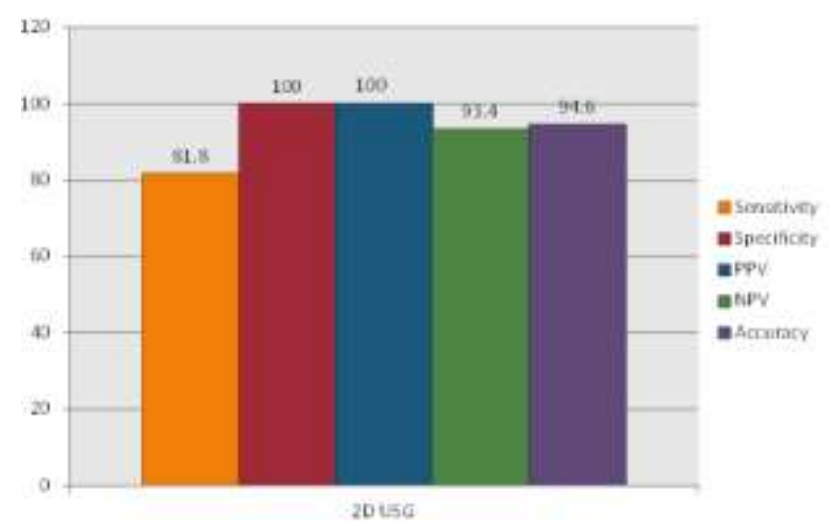

Figure 1: Diagnostic utility of 2D-USG for detecting MDAs in total study sample $(n=75)$.
If we take into account our total study sample of 75 patients then 2D USG detected 18 cases of MDA while MRI detected 22 cases of MDA. In 64 patients of infertility (both primary infertility + recurrent abortion) MDA was detected in 18 cases on MRI and 14 cases on 2D USG (Table 6).

So 2D transabdominal USG failed to detect 04 cases of MDA in total study population bringing overall sensitivity of 2D USG as $81.8 \%$ [59\%-94\% with $95 \%$ confidence interval (CI)] vis a vis $100 \%$ of MRI. However the specificity of 2D USG is $100 \%$ [92\%-100\% with 95\% CI] which is comparable with MRI. The PPV of 2D USG is of $100 \%$ [78\%-100\% with $95 \% \mathrm{CI}$, NPV is $93.4 \%[82 \%-98 \%$ with $95 \% \mathrm{CI}]$ and accuracy is $94.6 \%$ [86\%-98\% with 95\% CI] (Table 7, Figure 1). 
All these figures are highly significant statistically (Fisher exact and Chi Square tests) with $\mathrm{p}<0.000$.

The table 8 shows the compiled figures for sensitivity, specificity, PPV, NPV and accuracy of two dimensional abdominal ultrasonography (2D USG) for various sub groups and subtypes and total study sample (Table 8). All these figures are highly significant statistically (Fisher exact and Chi-square tests) with $\mathrm{p}<0.000$.

17 year old unmarried female presented with primary amenorrhoea. No other significant history. She underwent two dimensional transabdominal ultrasonography (2D USG) and MRI of the pelvis

Figure 2 and 3: 30 year old married for 04 years presented with primary infertility. Her menses were irregular with scanty bleeding. She underwent two dimensional transabdominal ultrasonography (2D USG) and MRI of the pelvis.

2D USG: (true coronal and sagittal plain) Normal appearance of fundus, uterine cavity, cervix and both ovaries (Figure 2).

MRI: (T2 TSE axial and true coronal plain) uterine cavity showing a gentle curve towards left side with single cornua on left. Fundus is small. No rudimentary horn noted. Bilateral ovaries are normal (Figure 3).

Diagnosis: 2D USG-Normal scan; MRI: Unicornuate uterus (AFS type II d) without rudimentary horn.

Figure 4 and 5: 40 year's old female married for 17 years presented with recurrent abortions. She had 3 abortions in the last 17 years with POG $<20$ weeks each time. Her menses were irregular. She underwent two dimensional transabdominal ultrasonography (2D USG) and MRI of the pelvis

2D USG: (axial and true coronal plain) uterine cavity is bifid with a septum originating from fundus and continuing up to mid part of uterine cavity. External contour of fundus is normal. Cervix is normal. Bilateral ovaries are normal (Figure 4).

MRI: (T2 TSE axial and true coronal plain) External fundal contour is normal with a septum originating from its inner part and dividing the upper part of uterine cavity in two parts. The septum has a sharp inferior part. Cervix is normal. Bilateral ovaries are normal (Figure 5).

Diagnosis: Septate uterus with incomplete septum (AFS type $\mathrm{V} \mathrm{b}$ ) on both 2D USG and MRI.

Figure 6 and 7: 17 year old unmarried female presented with primary amenorrhoea. No other significant history. She underwent two dimensional transabdominal ultrasonography (2D USG) and MRI of the pelvis.
2D USG: (axial and true coronal plain) There is a deep fundal cleft $(>1 \mathrm{~cm}$ deep) with widely separated uterine horns. Cervix not adequately visualised. Endometrium is thin and midline. Bilateral ovaries are normal (Figure 6).

MRI: (T2 TSE axial and true coronal plain) There is duplication of uterus and cervix with wide separation of the two uterine horns. A deep fundal cleft ( $>1 \mathrm{~cm}$ deep) is noted extending down with two separate cervices. Bilateral ovaries are normal (Figure 7).

Diagnosis: 2D USG: Uterus didelphys D/D: Bicornuate uterus; MRI: Uterus didelphys (AFS type III).

2D USG: (axial and sagittal plain) Uterine and cervix not visualised. Vagina visualised in the lower part .Both ovaries are normal (Figure 8).

MRI: (axial and sagittal plain) Uterus and cervix not visualised. Lower part of vaginal visualised. Bilateral ovaries are normal (Figure 9).

Diagnosis: Complete uterine agenesis (AFS type I: Mayer Rokitansky Kuster Hauser syndrome- Type A) on both 2D USG and MRI.

\section{DISCUSSION}

Mullerian duct anomalies result from defects arising during development of female reproductive organs. Since Mullerian ducts are embryologically distinct from female gonads i.e. ovaries, the patient with Mullerian duct anomalies have normal ovaries. ${ }^{13}$ The diagnosis of MDAs occurs at the time of onset of menses when a female child fails to attain menarche or after marriage when the lady fails to conceive and during infertility workup the MDA is diagnosed. Sometimes the females present with recurrent abortions in which MDAs are a cause of recurrent abortion needs. Diagnosis of MDAs and their sub type identification is important for management of these structural anomalies. The surgical approach for correction of these disorders differs completely for disorders which may look similar on imaging. So correct identification and description of MDA is a vital component for planning appropriate treatment.
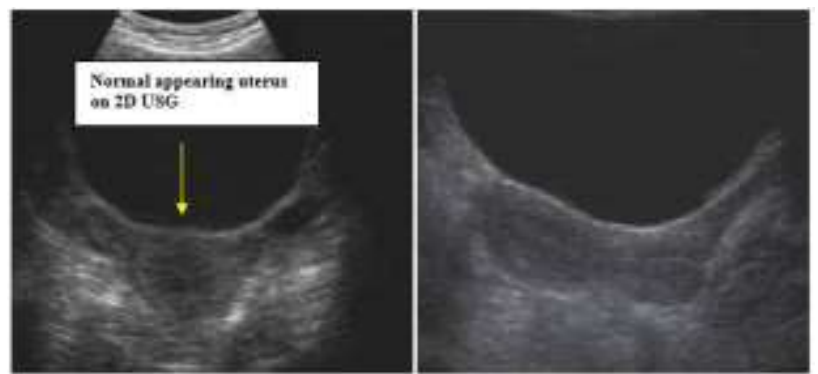

Figure 2: 2D USG pelvis showing images in true coronal and sagittal pain. 


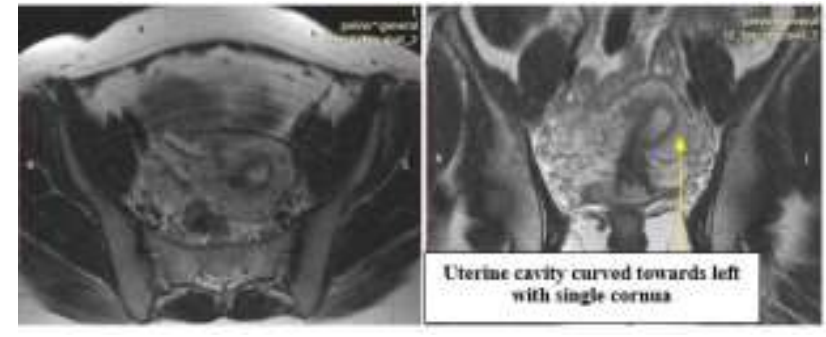

Figure 3: MRI pelvis showing images in T2WI axial and true coronal pain.

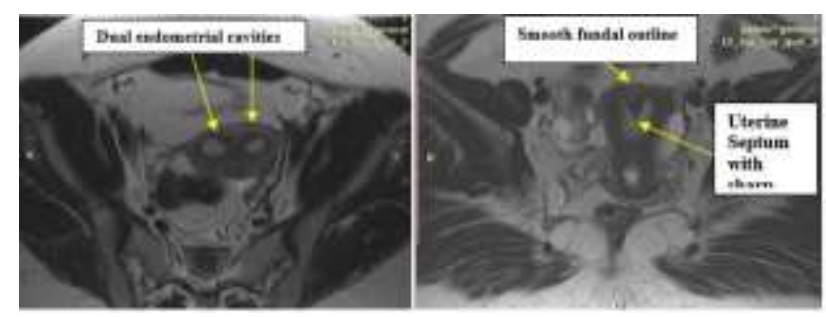

Figure 4: 2D USG images of pelvis in axial and true coronal pain.

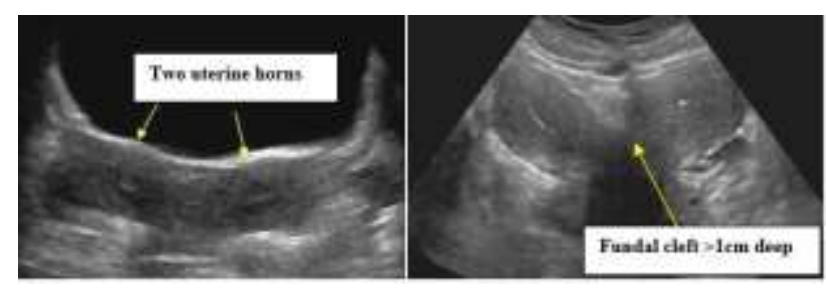

Figure 5: MRI images of pelvis in T2WI axial and true coronal pain.



Figure 6: 2D USG images of pelvis in axial and true coronal pain.

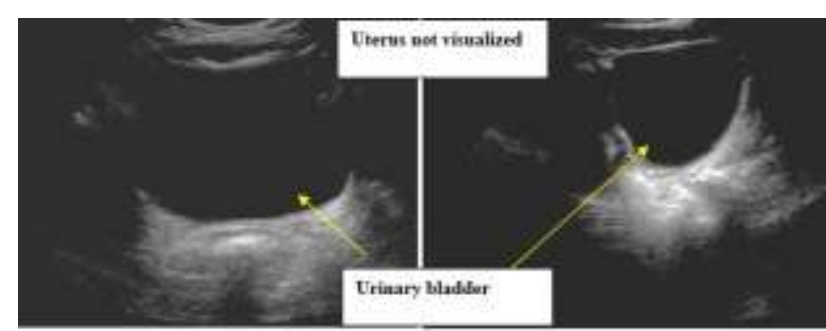

Figure 7: MRI images of pelvis in T2WI axial and true coronal pain.

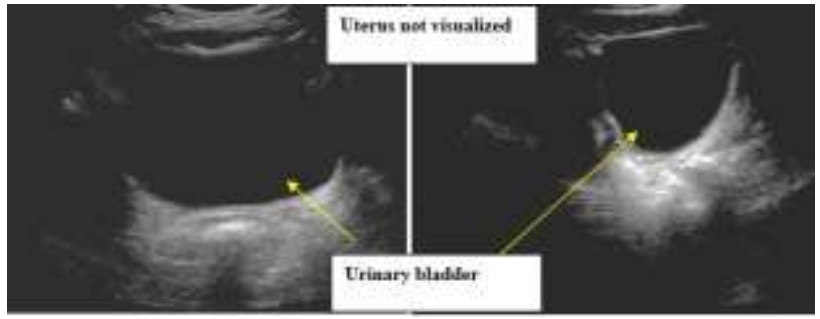

Figure 8: 2D USG images of pelvis in axial and sagittal pain.

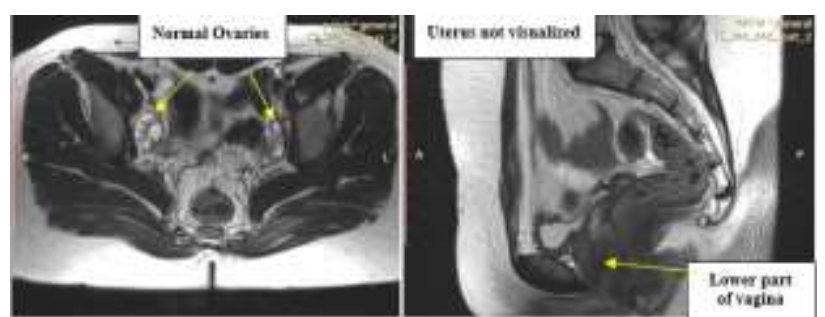

Figure 9: MRI images of pelvis in T2WI axial and true coronal pain.

With the advent of advanced high resolution ultrasonography scanners, it is often considered first imaging option in females in which there is clinical suspicion of MDAs. Both 2D USG as well as 3D USG are used to evaluate females with suspected MDAs. Ultrasonography being simple and widely available without radiation risk repeated examinations can be performed.

However there are limitations with ultrasonography in the form of inadequate visualization of uterus because of multiple factors like obesity, bowel gas and uterine position in pelvis. Another very important limitation is inter-operator variability where the expertise and experience of the operator plays an important role. An experienced operator can make a huge difference in the outcome by giving better diagnostic information even with 2D USG. So an adequately trained operator is an important requirement with 2D USG. At times it is difficult even for a well trained and experienced operator to adequately visualize the external fundal contour.

In this study we have used transabdominal ultrasonography as the mode of imaging for pelvis. This is done because our sample consists of patients with primary amenorrhea who were unmarried and doing transvaginal scan in this patient group was not acceptable. So to attain uniform imaging standards in our study population we have performed ultrasound only by transabdominal route.

The prevalence of MDA in our total study population is $29.3 \%$. The prevalence of MDAs in patients of infertility in our study population is $25 \%$ which is higher as compared to MDAs prevalence of $8 \%$ in infertility patients published by Human reproduction update 2011 . $^{14}$ 
This could be due to smaller sample size of the study population of 75 as the compared to Human reproduction update 2011 by Chan et al, where the sample size comprised of 89,861 cases. $^{14}$

The prevalence of MDAs in our population of recurrent abortions/miscarriages is $37.5 \%$ which is again high as compared to prevalence of $13.3 \%$ mentioned in Human Reproduction Update 2011. This could again be attributable to the smaller sample size.

The prevalence of MDAs in patients with primary amenorrhea in our study is $54.5 \%$. In patients of primary amenorrhea most common Mullerian duct anomaly is uterine aplasia and hypoplastic uterus with prevalence rates of both being $18.1 \%$. Similar prevalence was recorded in study done by Rao and Pillai who performed a study with a sample size of 40 to evaluate causes of primary amenorrhea and found a prevalence of MDAs in $50 \%$ of study their sample. Another study done by Kumar and Mittal on a study sample of 48 patients to evaluate etiological factors for primary amenorrhea has revealed prevalence of MDAs as $54.2 \%$ in their study population. $^{15,16}$

The most common MDA present in our total study group of 75 patients is arcuate uterus with prevalence of $8 \%$. Similar finding was reported in Human reproduction update 2011 where the most common MDA is arcuate uterus with a prevalence of $3.9 \%$.

Second most common anomaly is unicornuate uterus with prevalence of $6.7 \%$, followed by septate and bicornuate uterus with prevalence of $4.2 \%$ each.

Among patients of primary infertility the most common MDA is arcuate uterus with a prevalence rate of $10.4 \%$. Though arcuate uterus is the most common Mullerian duct anomaly seen in patient with infertility but its prevalence is not significantly different from that of general population. This has been validated in Human reproduction update 2011 .

In our study, amongst patients with recurrent abortions most common Mullerian duct anomaly is unicornuate uterus with a prevalence of $18.7 \%$. This finding is different from the Human Reproduction Update 2011 where the most common cause of recurrent abortions is septate uterus. This again could be attributed to the smaller sample size in our study population. However, a study done by Fedele and Bianchi confirms the poorest reproductive outcome in unicornuate uterus. ${ }^{17}$ Another study by Rock JA confirms poorest fetal survival in pregnant patients with unicornuate uterus. ${ }^{18}$

In two of our cases in the study we found a small hypoplastic uterus closely abutting the posterior surface of urinary bladder. There is no demarcation between endometrium and myometrium both on 2D USG and MRI. In one of our cases who presented with primary amenorrhea and pain abdomen, 2D USG was able to identify the bifid uterine cavity with fundal cleft measuring $>1 \mathrm{~cm}$ and a hypoechoeic collection in its lower cervical part. On MRI the findings of $2 \mathrm{D}$ USG were confirmed, there is bicornuate uterus with partial vaginal agenesis in upper part of vagina and the cervix containing blood degradation products.

In our study out of 11 cases of primary amenorrhoea both 2D USG and MRI diagnosed 06 cases of MDAs with no discrepancy in findings.

The diagnosis of unicornuate uterus on 2D USG is arrived on the basis of curved uterine cavity to one side and inability to trace the endometrial cavity to other cornua. In our study we encountered two patients with rudimentary horns and one of these cases also has a functional endometrial tissue within. These rudimentary horns were abutting the uterus and suspicion of rudimentary horn with unicornuate was raised in view of the clinical suspicion of Mullerian duct anomaly. So on 2D USG the presence of rudimentary horn may be helpful to identify unicornuate uterus or may raise a suspicion of unicornuate uterus. ${ }^{19,20}$ In two cases 2 D USG failed to diagnose unicornuate uterus as there were no rudimentary horns and reported them as normal.

2D USG could diagnose only 03 cases of unicornuate uterus as compared to 05 cases diagnosed by MRI. Thus 2D USG has sensitivity of $60 \%$, specificity of $100 \%$, PPV of $100 \%$, NPV of $97.3 \%$ and accuracy of $97 \%$. 2D USG performed poorly in detecting unicornuate uterus as compared to MRI.

In our study we have encountered one case of uterus didelphys with widely displaced uterine horns and duplication of the cervices which were appreciated on 2D USG. Duplication of the vagina was not apparent. MRI of the same patient demonstrated two widely divergent uterine horns and two widely separate cervices. Vaginal duplication could not be ascertained even on MRI. Based on the imaging findings diagnosis of uterus didelphys is considered. So in this case 2D USG scored as good as MRI in diagnosing uterus didelphys.

In bicornuate uterus there is some degree of fusion between two Mullerian ducts thus differentiating this entity from uterus didelphys where there is complete separation of two Mullerian ducts without any evidence of fusion. In our study 2D USG has diagnosed 04 cases of bicornuate uterus but only 03 cases were confirmed on MRI, one case of septate uterus was falsely diagnosed as bicornuate uterus. So the 2D USG has sensitivity of $100 \%$, specificity of $98.6 \%$, PPV of $95 \%$, NPV of $100 \%$ and accuracy of $99 \%$ in diagnosing MDAs as compared to MRI.

In our study two cases of septate uterus were diagnosed comfortably by 2D USG, however in the third case it failed to characterise the fundal contour correctly and 
misdiagnosed it as bicornuate uterus. This reinforces the requirement to visualise the fundal contour in true coronal plane and its correct interpretation which at times is difficult with 2D USG. So, 2D USG has a sensitivity of $66.6 \%$, specificity of $100 \%$, PPV of $100 \%$, NPV of $98.6 \%$ and accuracy of $99 \%$ in detecting MDAs as compared to MRI.

In our study 2D USG diagnosed 04 cases of arcuate uterus and missed two cases which were picked up on MRI. Thereby 2D USG has a sensitivity of $66.6 \%$, specificity of $100 \%$, PPV of $100 \%$, NPV of $97.3 \%$ and accuracy of $97.2 \%$ in detecting MDAs as compared to MRI.

Based on the results of our study it is inferred that two dimensional transabdominal ultrasonography has low sensitivity $81.8 \%$ combined with high specificity $100 \%$ (except for bicornuate uterus where specificity is $98.6 \%$ ) for detecting MDA. So the cases which were positive on 2D USG have a high probability of having MDAs. Similar findings are found in a study done by Nicolini $U$ et al (in the year 1987), in which he evaluated the role of 2D USG via transabdominal route in screening for uterine malformations. ${ }^{21}$ He found a sensitivity of $42.9 \%$ and specificity $97.8 \%$ in detecting uterine malformations. But the sensitivity in detecting MDAs with 2D USG in the study by Nicolini et al is much less as compared to our study. This could be attributed to the non-availability of good resolution ultrasonography imaging at that time. However the sample size of Nicolini et al was 89 which were slightly more than our study sample of 75 patients.

However, amongst MDAs where fundal contour is sub optimally visualised 2D USG seems to have poor specificity for differentiating bicornuate from septate uterus as compared to MRI. Similarly, on 2D USG it can be confusing to differentiate bicornuate bicollis uterus from uterus didelphys. In one of our case on 2D USG, we found a bifid uterine cavity with a deep fundal cleft which was extending till lower part of uterine cavity. There are two separate cervical canals noted. MRI was done in this case and it showed fusion of bifid uterine cavity in lower part of uterus confirming diagnosis of bicornuate bicollis uterus.

In our study, patients with primary amenorrhoea who had uterine agenesis and hypoplasia were diagnosed by 2D USG with results comparable to MRI. It is because of accurate interpretation of absence of uterus and cervix or its small hypoplastic variant in the presence of normal ovaries which were picked up on 2D USG.

Adequate distension of urinary bladder is key to image uterus and its external contour in various planes. Both over and under distension hampers adequate assessment of uterus on pelvic ultrasonography.

Trans Vaginal Ultrasonography (TVS) and three dimensional ultrasonography (3D USG) are better than two dimensional transabdominal ultrasonography (2D USG) in their ability to image uterus in true coronal plane. Imaging uterus in coronal plane provides information about fundus which is vital in characterising various sub types of MDAs. Few patient related factors like obesity, bowel gas shadows and abnormal position of uterus like retroverted uterus which also contribute to reduced sensitivity and specificity in assessing MDAs by 2D USG as compared to MRI.

\section{CONCLUSION}

This study is unique and is the only study which has taken into account all the variants of Müllerian duct anomalies in one study. Most common MDA in total study sample and in primary infertility group is arcuate uterus while in recurrent abortions group it is unicornuate uterus. The study is in agreement with previous studies that 2D USG has high specificity coupled with low sensitivity for detecting MDAs. When used in isolation 2D USG is able to identify major uterine anomaly easily but it may not be able to sub classify it in all cases due to its imaging restrictions. Sometimes it may entirely miss a Mullerian duct anomalies due to limitations mentioned above.

\section{ACKNOWLEDGEMENTS}

Authors would like to thank Dr. Kapil Semalti, Dr. Akshay Kambli.

Funding: No funding sources

Conflict of interest: None declared

Ethical approval: The study was approved by the Institutional Ethics Committee

\section{REFERENCES}

1. Ashton D, Amin HK, Richart RM, et al. The incidence of asymptomatic uterine anomalies in women undergoing transcervical tubal sterilization. Obstet Gynecol. 1988;72:28-30.

2. Byrne J, Nussbaum BA, Taylor WS. Prevalence of mullerian duct anomalies detected at ultrasound. Am J Med Genet. 2000;94:9-12.

3. Sotirios H. Saravelos KA. Prevalence and diagnosis of congenital uterine anomalies in women with reproductive failure: a critical appraisal. Human reproduction Update. 2008;5:415-29.

4. Carrington BM, Hricak H, Nuruddin RN. Mullerian duct anomalies: MR imaging evaluation. Radiology. 1990;176:715-20.

5. Fedele L, Dorta M, Brioschi D. Magnetic resonance evaluation of double uteri. Obstet Gynecol. 1989;74:844-7.

6. Raga F, Bonilla MF, Blanes J. Congenital mullerian anomalies: diagnostic accuracy of three-dimensional ultrasound. Fertil Steril. 1996;65:523-28.

7. $\mathrm{Wu} \mathrm{MH}, \mathrm{Hsu} \mathrm{CC}$, Huang KE. Detection of congenital mullerian duct anomalies using three- 
dimensional ultrasound. J Clin Ultrasound. 1997;25:487-92.

8. Canzone G. 2D-3D USG in diagnosis of Uterine malformations. Donald School J of USG in Obs and Gynae. 2007;1(3):77-9.

9. Nahum GG. Uterine anomalies. How common are they, and what is their distribution among subtypes? J Reprod Med. 1998;43(10):877-87.

10. Robbins JB, Parry JP, Guite KM, MRI of PregnancyRelated Issues: Mullerian Duct Anomalies. AJR. 2012;198:302-10.

11. Robert NT, McCarthy SM, Mullerian Duct Anomalies: Imaging and Clinical Issues. RSNA, Radiology. 2004;233:19-34.

12. Fedele L, Dorta M, Brioschi D. Magnetic resonance evaluation of double uteri. Obstet Gynecol. 1989;74(6):844-7.

13. Speroff L, Glass RH, Kase NG. Development of the mullerian system. In: Mitchell C, ed. Clinical gynecologic endocrinology and infertility. 6th ed. Baltimore, Md: Lippincott, Williams and Wilkins. 1998;124.

14. Chan YY, Jayaprakasan K, Zamora J. The prevalence of congenital uterine anomalies in unselected and high-risk populations: a systematic review. Hum Reprod Update. 2011;17(6):761-71.

15. Rao K, Pillai NV. Primary amenorrhoea: analysis of 40 cases. J Indian Med Assoc. 1991;89(2):42-3.
16. Kumar S, Mittal S. Primary amenorrhea: analysis of 48 cases. J Indian Med Assoc. 1998;96(4):119-20.

17. Fedele L, Bianchi S, Tozzi L, et al. Fertility in women with unicornuate uterus. $\mathrm{Br} \mathrm{J}$ Obstet Gynaecol. 1995;102:1007.

18. Rock JA. Surgery for anomalies of the mullerian ducts. Tompson JD, Rock JA, eds. TeLind's Operative Gynecology. 9th ed. Philadelphia, Pa: JB Lippincott Williams and Wilkins. 2003:705.

19. Pellerito JS, McCarthy SM, Dovie MB. Diagnosis of uterine anom- alies: relative accuracy of MR imaging, endovaginal sonography, and hysterosalpingography. Radiology. 1992;183:795800.

20. Brody JM, Koelliker SL, Frishman GN. Unicornuate uterus: imaging appearance, associated anomalies and clinical implications. AJR Am J Roentgenol. 1998;171:1341-7.

21. Nicolini U, Bellotti M, Bonazzi B. Can ultrasound be used to screen uterine malformations? Fertil Steril. 1987;47:89-93.

Cite this article as: Senger KPS, Singh AK, Sharma V, Singh A, Ravikumar R. Detection of mullerian duct anomalies: diagnostic utility of two dimensional ultrasonography as compared to magnetic resonance imaging. Int J Reprod Contracept Obstet Gynecol 2017;6:20-8. 